\title{
The Design of an Intelligent Security Access Control System Based on Fingerprint Sensor FPC1011C
}

\author{
Yan Wang ${ }^{1,2}$, Hongli Liu ${ }^{1}$, Jun Feng ${ }^{2}$ \\ ${ }^{1}$ College of Electrical and Information Engineering, Hunan University, Changsha, China \\ ${ }^{2}$ College of Electrical Engineering, Nanhua University, Hengyang, China \\ E-mail: wangyan5406@163.com \\ Received April 29, 2010; revised May 30, 2010; accepted June 5, 2010
}

\begin{abstract}
This paper deals with the design of an intelligent access control system based on the fingerprint sensor FPC1011C. The design uses the S3C2410 and TMS320VC5510A as the system processor. A fingerprint acquisition module and a wireless alarm module were designed by using the fingerprint sensor FPC1011C and GPRS module SIM100 respectively. The whole system was implemented wireless alarm through messages and GPRS-Internet in the GSM/GPRS web. In order to achieve the simple and high Real-time system, the $\mu \mathrm{C}$-Linux system migration was also implemented.
\end{abstract}

Keywords: Fingerprint Sensor, Security Access Control System, ARM, Wireless Alarm

\section{Introduction}

Generally, traditional fingerprint access control system is based on the computer or microcontroller. The Computer platform is hard to carry because of high cost and the MCU platform is more difficult or even impossible to achieve for its features such as data processing capabilities and storage capacity constraints. The circuit of wireless alarm is vulnerable and security is not high with high maintenance cost; special networks of wireless alarm are too costly. All those factors prevent the development of fingerprint access control system.

This design uses ARM + DSP microprocessor and advanced fingerprint sensor FPC1011C, and is also equipped with GPRSIGSM network for wireless alarm. In the Fingerprint access control system, it is lack of control using DSP microprocessor [1] and weak processing capability using ARM microprocessor [2]. And thus it may be an attractive approach to a more perfect framework for fingerprint access controlling by combining ARM and DSP in an integrative platform. Fingerprint sensor FPC1011C is suitable for both dry and wet fingers due to its adopted reflective detection technology. As a public network with wide coverage, GPRS/GSM supports real-time transmission, lower operation costs, SMS service and internet access [3]. So it can be widely used in wireless alarm of access control system.

\section{System Principles}

System principles are as shown in Figure 1. The system acquires fingerprint data from the fingerprint device. Then it will be sent to the DSP processor, where fingerprint data are processed and effective features are extracted. In ARM processors, the feature will be registered to the database or acts as data for triggering target event: if the fingerprint's feature and some sample in the database matches, access is permitted, or there will be alarm [4]. Thereinto, alarms are divided into local alarms and wireless ones. Local alarm is given by sound and light lamps. Wireless alarm send messages to the concerned mobile phone by GPRS module through which connect GPRS/ GSM network controlled by ARM, and it is also sent to the PC alarm monitoring center by GPRS-internet network.

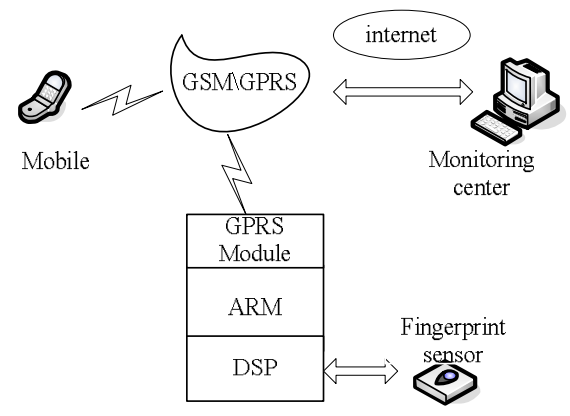

Figure 1. System principle. 


\section{Hardware Design}

The system hardware includes: DSP, fingerprint sensor, ARM controller, GPRS module and its external power supply module, extended memory devices、 LCD and keyboard.

\subsection{DSP, FPC1011C and ARM}

This design uses a DSP chip TMS320VC5510A as the processor and the most advanced fingerprint sensor capacitance FPC1011C. Control chip used ARM Samsung S3C2410, which is a 16/32-bit embedded RISC microprocessor [5] based on ARM920T core. The interface of ARM + DSP and FPC1011C is shown in Figure 2.

FPC 1011C is a capacitive fingerprint sensor surface shape which is launched successfully by Finger Prints, a Swedish company [6]. It has high image quality, wear resistance, static and low power consumption. It uses the reflective detection technology which is a patent by Finger Prints. The electrical pulse signal is generated form the internal IC, and then passed to finger through the conductive ABS frame. Because the human body is a conductor, fingerprints will produce high and low voltage form Valley and Ridge when the pulse through the finger. The wafer body accepts and amplifies the signal, then exports digital signal of fingerprint after $\mathrm{A} / \mathrm{D}$ conversion. Because of its unique detection technology, it has good applicability of the dry and wet fingers and longer life.

FPC1011C provides a high-speed SPI interface to communicate with the DSP processor TMS320VC5510A, so its hardware interface circuit design is simple with fast transfer rate. DSP's Mcbsp can simulate the SPI protocol, complete with a seamless interface to the fingerprint sensor. The useful pins of FPC1011C are CS_N, SO, SI, SCK and so on. CS_N is the chip select signal line, which is directly to ground, so that the fingerprint sensor has been in the selected state. SO and SI is the SPI data lines which connected with DSP-DR2, DX2 to transmit the serial data. SCK is the clock signal line and linked by DSP's CLKX2 to provide the sampling clock [7].
TMS320VC5510A host interface (HPI) is an interface designed by TI Company can greatly simplify the hardware design of DSP exchanging data with external device. Communication can be achieved by linking ARM's I/O port to the DSP's HPI. System maps the all HPI interface controllers, address registers, data registers which have unified address to the S3C2410's I/O memory space. As the basic HPI, HCS enables input signal, and controls HPI data transfer with HDS1, HDS2. Address line A1 A4 produces the required control signals by HPI.

\subsection{GPRS Module}

The design of GPRS module uses SIM100, and it has high performance with enhanced AT command set. The technical specifications are suitable for the development of GPRS-based wireless products, and it provides users with a fully functional system interface, saving users time and cost of development. Module supports an external SIM card, and you can connect directly with the SIM card. The module can automatically detect and adapt to SIM card type, which have GPRS service. Module supports GSM, SMS business and the GPRS access internet [8]. GPRS and SIM interface circuit diagram are shown in Figure 3.

\section{System Software}

\section{1. $\mu$ CLinux Transplantation}

Linux operating system as a derivative $\mu$ Clinux, followed the vast majority of linux features, overcame the shortage of cell-free MMU, and had powerful network processing capabilities, it also can support various file systems and has became mainstream in embedded operating system. This design transplants the ARM- $\mu$ CLinux, and the specific process includes the development of environment, preparation, revision of Bootloader, modification and compilation of the kernel source, transplantation of kernel image and the file system [9].

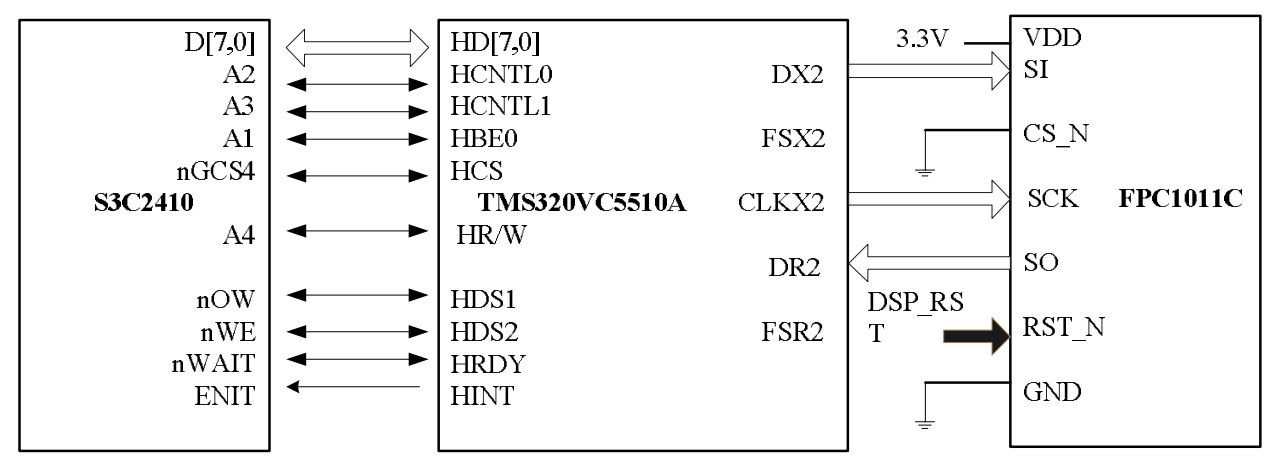

Figure 2. Interface of ARM + DSP and FPC1011C. 


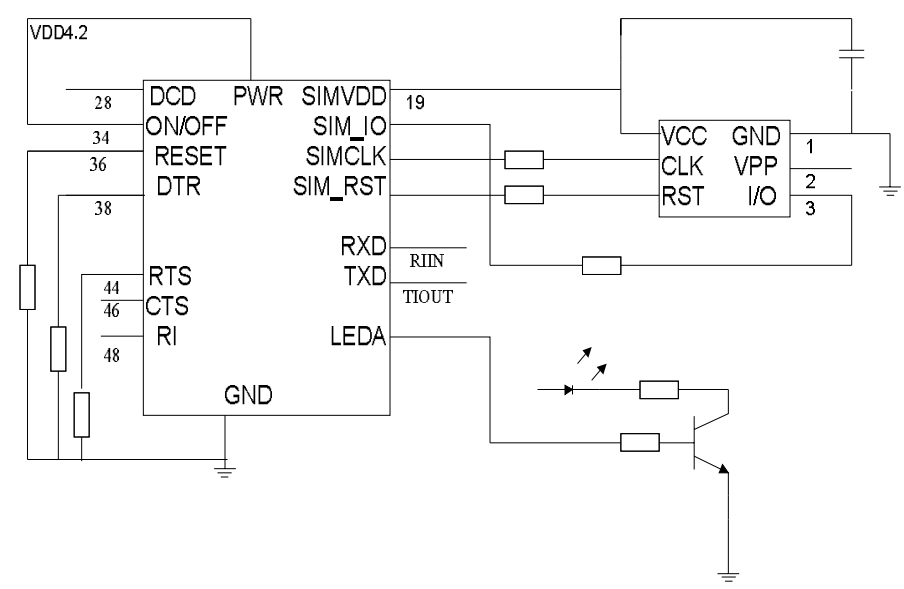

Figure 3. Interface of SIM100 with the SIM Card.

\subsection{GPRS Driver Design}

GPRS module driver includes initializing, opening, closing and sending or receiving data [10]. By calling GPRS_ Init() function to achieve the basic GPRS module initialization, includes initializing serial port, testing signal quality, opening AT protocol and GPRS, setting IP address, port number and so on. It is concentrated by calling the AT command, AT + WIPDATA and AT + WIPCLOSE order to achieve the connection beginning and closing. S3C2410 communicates with GPRS module by AT command. GPRS_SendData() is used to send AT commands to the GPRS module, and GPRS_RecvData() accepts GPRS data module. GPRS_SendMsg() sends a short message, and GPRS_RecvMsg() parses text messages.

System is mainly divided into two parts: the fingerprint processing and ARM processor control.

1) The main program flow chart of fingerprint processing is shown in Figure 4. Firstly, initialization operation, includes the DSP system initialization and peripheral initialization; and then it starts to test whether it has fingerprints input on the fingerprint sensor. If there are fingerprints input on the fingerprint sensor, it identifies this signal. The fingerprint image starts preprocessing work after recognition. This fingerprint image quality evaluation is in front of the pretreatment on the identification of incoming, if the quality of fingerprint is good enough then continue the rest of the pretreatment. If image quality is poor, this fingerprint data is discarded and required to identify a fingerprint data again. After the extraction of high-quality fingerprint image feature, the judge is the registered fingerprint or fingerprint match. If it is to register the fingerprint, the fingerprint data is put into the database. If it is to match the fingerprint, system will compare the fingerprint with each sample in the library, which leads to a result means weather or not match successfully.
2) External LCD of ARM provides user interface and prompts information, the keyboard can input data [11]. Access Administrators use them to set the system to perform specific technical indicators [12], for example, opening access, allowing access to the total number, etc, accessing to personnel information prompted by operating the input fingerprint. The chart of ARM processor to control the main program flow is shown in Figure 5.

Firstly, initializing the module, and then determining whether there is an order input. If there is administrator command, prompting for a password, and the fingerprint verification follow-up after the administrator can operate. If there is ordinary user command, the user will be reminded to input the password and fingerprint, the access control will be lifted after authentication. Otherwise, the control alarm module will conduct wireless alarm.

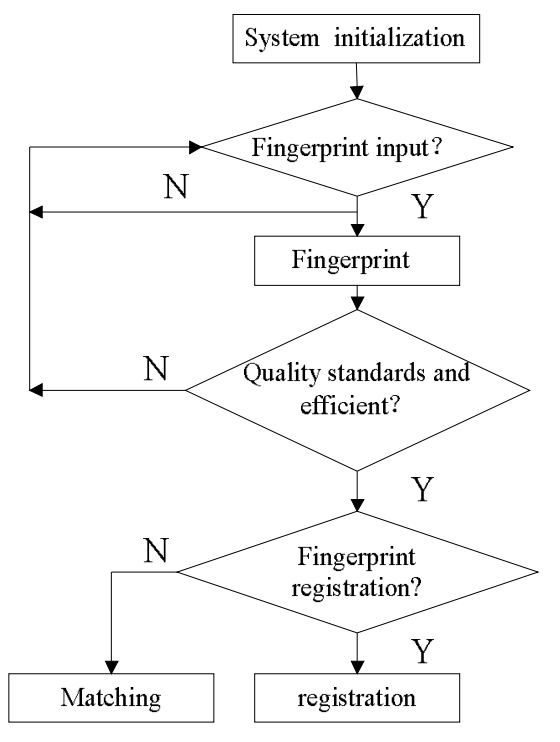

Figure 4. Flow of fingerprint procedures. 


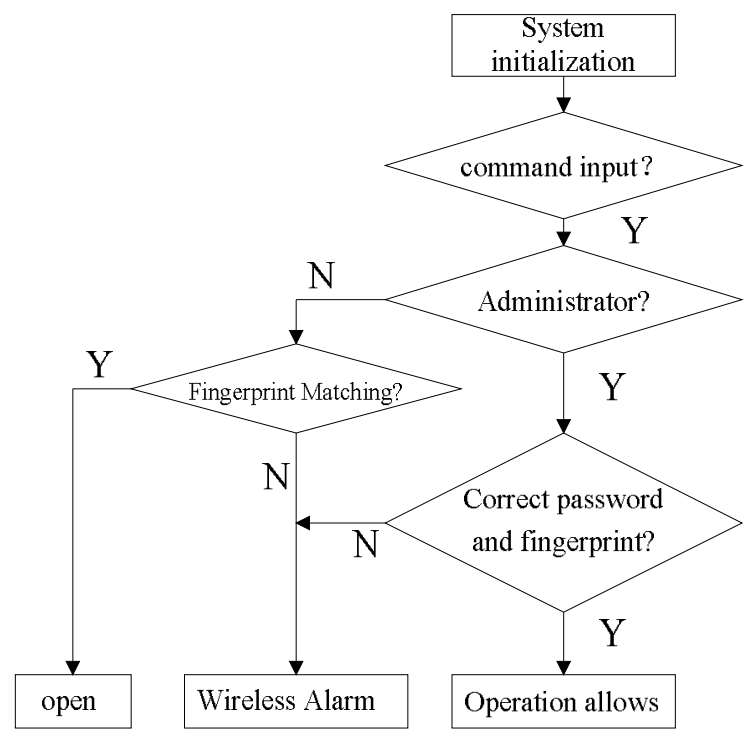

Figure 5. Flow of ARM control.

\section{Conclusions}

In this paper, we researched the embedded wireless alarm system and fingerprint access control, which use the $\mathrm{ARM}+\mathrm{DSP}$ dual processors to improve system control and data processing capabilities, and it uses GPRS/GSM network to improve the speed and security of alarm and reduce the cost. After transplanting $\mu$ CLinux system, system function can be improved and extended cut, which make the system has good openness. This design is efficient, compact, and with low power cost and high safety level, which significantly meet the requirements of the current fingerprint access control system and increased the levels of Access Control System.

\section{References}

[1] K. L. Zhou and Z. X. Lu, "Design of Vehicle Locks Based on DSP and Fingerprint Identification System,"
Journal of Shanxi University of Science \& Technology, Vol. 27, No. 5, 2009, pp. 103-105.

[2] C. G. Xie, "ARM-Based Automatic Fingerprint Identification System," Microcomputer Information, Vol. 25, No. 1-4, 2009, pp. 292-294.

[3] H. Guo, Y. S. Guo and Y. Chen, "The Implementation of Remote Meter Reading System Based on Linux and GPRS," Application of Electronic Technique, Vol. 34, No. 11, 2008, pp. 82-84.

[4] F. Ding, "ARM-Based Fingerprint Identification System Research and Implementation of [D]," South China University of Technology, Guangzhou, 2007.

[5] Z. M. Ma and Y. H. Xu, "ARM Based Embedded Processor Architecture and Application," Beijing University of Aeronautics and Astronautics Press, Beijing, 2002.

[6] J.-M. Nam, S.-M. Jung, D.-H. Yang and M.-K. Lee, "Design and Implementation of $160 \times 192$ Pixel Array Capacitive-Type Fingerprint Sensor," Circuits, Systems \& Signal Processing, Vol. 24, No. 4, 2005, pp. 401-413.

[7] L. Zhang, "Based on DSP and RF Card Embedded Fingerprint Identification System Design and Implementation of [D]," University of Electronic Science and Technology of China, Chengdu, 2009.

[8] K. Al-Begain, I. Awan and D. D. Kouvatsos, "Analysis of GSM/GPRS Cell with Multiple Data Service Classes," Wireless Personal Communications, Vol. 25, No. 1, 2003, pp. 41-57.

[9] Y. Li, "MCLinux Based on ARM Embedded System Theory and Application," Tsinghua University Press, Beijing, 2009.

[10] J. K. Zhang and X. Q. Zhang, "Embedded Linux System Development Technology Xiangjie-Based on ARM," Posts \& Telecom Press, Beijing, 2006.

[11] M. F. Du, "Development of Ethernet Interface for Smart Entry Controller Based on ARM \& Linux Architecture," Computer Engineering, Vol. 33, No. 16, 2007, pp. 234236.

[12] C. J. Zhang, "System of Defending to Rob by Tailing Behind of Double Gate by Chip Microcomputer Control," Computer Engineering and Applications, Vol. 43, No. 5, 2007, pp. 79-81. 